\title{
Novel multiplex RT-PCR assay to detect BCR/ABL mRNA variants
}

\author{
K Shires, (D) A Rust \\ Division of Haematology, Department of Pathology, University of Cape Town and NHLS, Groote Schuur Hospital, South Africa \\ Corresponding author, email: karen.shires@uct.ac.za
}

\begin{abstract}
The detection of the $t(9 ; 22)$ translocation, which results in the formation of the BCR/ABL oncoprotein, in patients diagnosed with chronic and acute leukaemias, allows for the administration of highly effective tyrosine kinase inhibitors such as imatinib and nilotinib. For the effective management of these patients it is important to monitor for minimal residual disease, allowing early therapy intervention prior to haematological relapse. Currently this is achieved through very sensitive quantitative real-time PCR assays. Unfortunately, these assays are highly specific to the $B C R / A B L$ variant expressed: p210, p190 or p230 and require identification of the transcript type prior to selection of the correct monitoring assay. We have developed a novel multiplex $B C R / A B L$ variant PCR assay that can identify the variant in a single qualitative assay, creating both a cost and time effective way to identify the most appropriate monitoring methodology for each patient.
\end{abstract}

\section{Introduction}

$B C R / A B L$ is a novel oncogene that is formed by a reciprocal translocation involving the long arms of chromosomes 9 and 22 $(t(9: 22)(q 34: q 11)$, which results in the fusion of the break-point cluster region $(B C R)$ gene and the Abelson murine leukaemia viral oncogene homologue $1(A B L)$ gene, forming the characteristic Philadelphia chromosome $(\mathrm{Ph}+)$ (elongated chromosome 22). The $B C R / A B L$ oncogene on the $\mathrm{Ph}+$ chromosome gives rise to the oncoprotein $B C R / A B L$, a constitutively active tyrosine kinase involved in many signalling pathways, while the reciprocal $A B L /$ $B C R$ on chromosome 9 does not yield a protein product. ${ }^{1}$ The translocation is most commonly associated with the development of Chronic Myeloid Leukaemia (CML), ${ }^{2}$ a clonal stem cell disorder that is characterised by abnormal myeloid proliferation proceeding through three distinct phases: chronic, accelerated and blastic crisis. ${ }^{3,4}$ The $t(9 ; 22)$ event is usually reported to occur in $>95 \%$ of CML cases, ${ }^{3}$ however as the primary oncogenic event in CML is now known to be the synthesis and action of BCR/ABL, the lack of detection in some historical cases of CML is probably a detection methodology issue rather than representing a true alternate disease mechanism in these cases. This oncoprotein also plays a role in the pathogenesis of subtypes of two other leukaemias, namely: $\mathrm{Ph}+$ Acute Myeloid Leukaemia (AML) and $\mathrm{Ph}+$ Acute Lymphoid Leukaemia (ALL), however its role in these cancers is likely to be as a secondary event rather than the initiating event and is part of a much more complex pathogenesis picture. It has been reported that as many as $30 \%$ of adult $A L L$ cases are $\mathrm{Ph}+$, while $<5 \%$ of $A M L$ cases express the $\mathrm{BCR} / \mathrm{ABL}$ oncoprotein. ${ }^{5,6}$

Due to the unique nature of the $B C R / A B L$ oncoprotein in these diseases, targeted drugs belonging to the class of tyrosine kinase inhibitors (TKI) are now used to effectively treat CML and aid in the induction responses of both $\mathrm{Ph}+\mathrm{AML}$ and $\mathrm{ALL}$. Stable expression of $B C R / A B L$ mRNA transcripts in each diseased cell has also led to novel monitoring methods, allowing detection of minimal residual disease (MRD) at levels as low as $0.001 \%$ disease. However, detection and monitoring of mRNA transcripts is complicated by the fact that the $B C R / A B L$ translocation can occur at several places within the $B C R$ gene (all fused to exon 2 of $A B L$ ), resulting in the formation of different transcript variants, and it is essential that the variant/isoform type is known for the effective monitoring of treatment responses, due to the different methodologies required for each transcript type. The three major variants are $\mathrm{M}$-bcr variants (major), which involve the fusion of the first 13 or 14 exons of $B C R$ to exon 2 of $A B L$ and are known as e13a2 (b3a2) and e14a2 (b2a2) transcripts, or p210, referring to the size of the protein produced, $210 \mathrm{kDa}$; $\mathrm{m}$-bcr variant (minor), which is the e1a2 translocation, often referred to as p190, as a smaller $190 \mathrm{kDa}$ is translated; and $\mu$-bcr variant (micro), which contains 19 exons of $B C R$ fused to exon 2 of $A B L$ (e19a2), and produces the largest $B C R / A B L$ protein of $230 \mathrm{kDa}$, known simply as p230.7 Little is known about the clinical consequences of the different proteins, as, if there are differences, they are subtle, as they appear to have identical functions within the cell, fundamentally those of a constitutively activated and cytoplasmic-restricted $A B L$ protein. $C M L$ is most usually associated with the p210 translocation types (M-bcr), ${ }^{3}$ 2008 , but p190 and p230 variants have also been identified, with the p230 variant usually being associated with a high neutrophil count $^{3}$ and a few limited studies suggesting that the p190 CML variant (associated with increased monocytes) may have a more aggressive disease course. ${ }^{8,9} \mathrm{Ph}+\mathrm{ALL}$ is more commonly associated with the 190 protein isoform, with only $20 \%$ of $B C R /$ $A B L$ positive cases being p210 positive. ${ }^{6,10} \mathrm{Ph}+\mathrm{AML}$ cases, while rare, are predominately associated with the p210 transcript type, ${ }^{5}$ although $\mathrm{p} 190$ cases have been reported. ${ }^{11}$

Diagnosis and treatment of these BCR/ABL-positive diseases does not require the identification of the specific mRNA variant, as the simple presence of the $t(9 ; 22)$ translocation (detected 
via karyotyping or $B C R / A B L F(S H)$ is sufficient for diagnosis and initiation of TKI therapy. However, the identification of the specific mRNA variant is essential for the monitoring of the patients during therapy, as the quantitative reverse transcription PCR (QRT-PCR) assays are highly specific to each variant, and false negative results will be obtained if the wrong test is used. Unfortunately, as a diagnostic laboratory we have observed the limited requests for testing for the alternate transcripts, which in turn has resulted in several cases of incorrect diagnoses (when cytogenetics is not performed in addition to QRT-PCR) or poorly controlled disease due to the apparent lack of transcript (incorrect test requested). This is primarily due to confusion regarding the prevalence and thus relevance of testing for the alternate variants and the cost that is associated with testing for each variant, which currently involves single variant RT-PCR assays.

We aimed to simplify the important step of $B C R / A B L$ transcript identification, by designing a multiplex RT-PCR assay that is capable of detecting all mRNA $B C R / A B L$ variants (involving exon 2 of $A B L$ ), in a more cost-effective, single test format. Standardised guidelines have been recommended by the Europe Against Cancer (EAC) programme and others for the detection and quantification of the various $B C R / A B L$ translocations, for use in MRD monitoring. ${ }^{12-14}$ We combined the recommended primer sets for p190 (primers ENF402/ENR561) and p210 (primers ENF501/ENR561) transcript amplification to develop a single multiplex RT-PCR assay that is capable of simultaneously detecting all of the common $B C R / A B L$ transcripts, including $\mathrm{p} 210$, p190 and p230. We assessed the assay for specificity, accuracy and sensitivity to establish its applicability in the diagnostic environment

\section{Materials and methods}

\section{Multiplex BCR/ABL RT-PCR assay}

Total RNA was extracted from the white cell component of 5-10 ml EDTA PB or $5 \mathrm{ml} \mathrm{BM}$ (received on ice, within 36 hours) using TRIZOL (Invitrogen) or equivalent methodology. The following specific reaction conditions and reagents were carefully designed to generate optimal, robust amplification in the multiplex setting specifically. RT was performed as a two-step reaction, with step 1 being the RNA denaturation and annealing of the CDNA primer: 2 ug RNA ( $5 \mu \mathrm{l}$ maximum volume), 50 pmol Oligo dT ( $1 \mu \mathrm{l}), 1 \mu \mathrm{l}$ $10 \mathrm{mM}$ dNTP (aids in primer-binding) and nuclease-free water to $13 \mu \mathrm{l}$ final reaction volume, incubation at $65^{\circ} \mathrm{C}$ for $10 \mathrm{mins}$, followed by immediate incubation on ice (not cooling block) for 5 mins. Step 2 represents the cDNA synthesis: $1 \mu \mathrm{l} 0.1 \mathrm{mM}$ DTT, $0.5 \mu$ l RNAse Out (40 U/ $\mu$ l, Invitrogen), $1 \mu$ l SuperScript ${ }^{\mathrm{TM}}$ III (200 U/ $\mu$ l Invitrogen), $4 \mu \mathrm{l} 5 \mathrm{X}$ SuperScript ${ }^{\mathrm{TM}} \mathrm{RT}$ buffer, $0.5 \mu \mathrm{l}$ nuclease-free water and $13 \mu \mathrm{l}$ from step 1 . This provides an RT reaction $(20 \mu \mathrm{l})$ containing reagents at the final concentrations of $2.5 \mathrm{pmol} / \mu \mathrm{l}$ Oligo DT, $0.5 \mathrm{mM}$ dNTP, $5 \mathrm{mM}$ DTT, $20 \mathrm{U}$ RNAse inhibitor, $200 \mathrm{U}$ SuperScript ${ }^{\mathrm{TM}} \mathrm{III}$ and 10\% cDNA. The reaction was incubated as follows: $50{ }^{\circ} \mathrm{C}$ for 60 mins (cDNA synthesis), $70{ }^{\circ} \mathrm{C}$ for 15 mins (denatures SuperScript ${ }^{\mathrm{TM}}$ ), $4^{\circ} \mathrm{C}$ for 5 mins, then immediately on ice (important step to ensure secondary/tertiary structures are prevented).
The final multiplex $B C R / A B L P C R$ reaction contained the following ( $25 \mu \mathrm{l}$ final volume): $1.5 \mathrm{mM} \mathrm{MgCl}, 0.8 \mathrm{mM} \mathrm{dNTP}, 1 \mathrm{X}$ Amplitaq Gold buffer (Applied Biosystems), $2.5 \mathrm{U}$ Amplitaq Gold (Applied Biosystems), $0.4 \mathrm{pmol} / \mu \mathrm{l}$ ENF402, $0.4 \mathrm{pmol} / \mu \mathrm{l}$ ENF501,12 $0.4 \mathrm{pmol} / \mu \mathrm{l}$ ENR561 ${ }^{12}$ and $2 \mu \mathrm{l}$ CDNA. The final $A B L \mathrm{PCR}$ reaction contained the following: $1.5 \mathrm{mM} \mathrm{MgCl}, 0.8 \mathrm{mM} \mathrm{dNTP}$, 1X AmplitaqGold buffer (Applied Biosystems), $2.5 \mathrm{U}$ Amplitaq

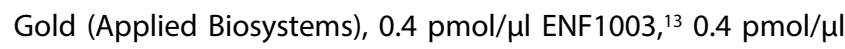
ENR $1063^{13}$ and $2 \mu \mathrm{l}$ CDNA. Primer sequences are shown in Table I. The multiplex PCR reactions are easily setup using pre-prepared mastermixes (MM) that can be stored at $-20^{\circ} \mathrm{C}$ in aliquots until required. Details of reagents that can be incorporated into a MM are shown in the supplementary data, as well as the simplified setup using these MM. The following PCR reaction conditions were applied: $95^{\circ} \mathrm{C}$ for 10 mins, 40 cycles of $94^{\circ} \mathrm{C}$ for $30 \mathrm{sec} /$ $65^{\circ} \mathrm{C}$ for $60 \mathrm{sec} / 68^{\circ} \mathrm{C}$ for $150 \mathrm{sec}, 68^{\circ} \mathrm{C}$ for 10 mins (using a Proflex PCR machine, with a ramp time of $0.5^{\circ} \mathrm{C} / \mathrm{secs}$ ). Amplicons were electrophoresed on a $3 \%$ TAE/agarose in 1X TAE for approx. 90 minutes at 80-100 v, stained with ethidium bromide and photographed under UV. DNA molecular ladders should be placed on either side of the samples to enable easier product sizing and a mixture of RNA containing p210 (e13a2) and p190 (e1a2) should be used as a positive control for the assay to ensure adequate separation between the $72 \mathrm{bp}$ and $90 \mathrm{bp}$ products.

Table I: Primer sequences used in the amplification of $B C R / A B L$ variants and control $A B L C D N A$

\begin{tabular}{lc}
\hline \multicolumn{1}{c}{ Primer name } & Primer sequence 5'-3' $^{\prime}$ \\
\hline ENF402 $^{12}$ & CTGGCCCAACGATGGCGA \\
ENF501 $^{12}$ & TCCGCTGACCATCAAYAAGGA \\
ENR561 $^{12}$ & CACTCAGACCCTGAGGCTCAA \\
ENF1003 $^{13}$ & TGGAGATAACACTCTAAGCATAACTAAAGGT \\
ENR1063 $^{13}$ & GATGTAGTTGCTTGGGACCCA \\
\hline
\end{tabular}

\section{Validation assessment}

A) Assay specificity was assessed by sequence analysis of the amplicons produced by the multiplex assay using commercial RNA samples (Invivoscribe) containing $B C R / A B L$ transcript types: e13a2 (IVS0003), e14a2 (IVS0011), e1a2 (IVS0032) and e19a2 (sequenced patient sample), as well as analysis of $B C R /$ $A B L$ negative RNA controls: IVS0035 (Invivoscribe), human testis RNA (Clontech), Jurkat/HL60/RPMI8226 RNA (cultured cell lines). Amplicons were excised from the multiplex assay agarose gel, purified using a standard PCR clean-up kit (Nucleobond) and the fragments sequenced using the ENR561 primer ${ }^{12}$ and the Bigdye Terminator cycle sequencing kit (Applied Biosystems), followed by separation on an ABI3500XI capillary analyser. Generated sequences were compared to the reference $B C R$ transcript sequence (NM_004327.5) and $A B L$ transcript sequence (NM_005157.5) to determine the break point in BCR and thus the transcript variant type. 
B) Accuracy of transcript variant calling was determined by analysis of 10 external quality control samples (NEQAS), in duplicate, in a technologist-blinded study using the multiplex RT-PCR assay and comparing the results to the official EQA reports.

C) The limit of detection for the assay was determined by analysing stored RNA that had previously been quantified using our SANAS accredited BCR/ABL p210 quantification assay, covering disease levels over 4 logs: $43.233 \%, 3.977 \%, 0.505 \%$, $0.050 \%, 0.005 \%$. These samples were reanalysed in duplicate on two separate occasions using the multiplex RT-PCR to determine the lowest level that could be readily detected.

\section{Results}

\section{Assay design}

This novel multiplex RT-PCR $B C R / A B L$ assay was designed to be able to identify $B C R / A B L$ transcript variants at diagnosis in a single simple assay, in patients suspected of suffering from $\mathrm{CML}, \mathrm{Ph}+\mathrm{AML}$ or Ph+ ALL. Briefly the multiplex assay should be performed as follows: RNA is extracted from either bone marrow (BM) or peripheral blood (PB) at diagnosis and a specialised RT is performed in duplicate. $B C R / A B L$ (primers ENF402/ENF501/ ENR561) and ABL (primers: ENF1003/ENR1063) reactions are then performed separately using a high fidelity Taq polymerase,
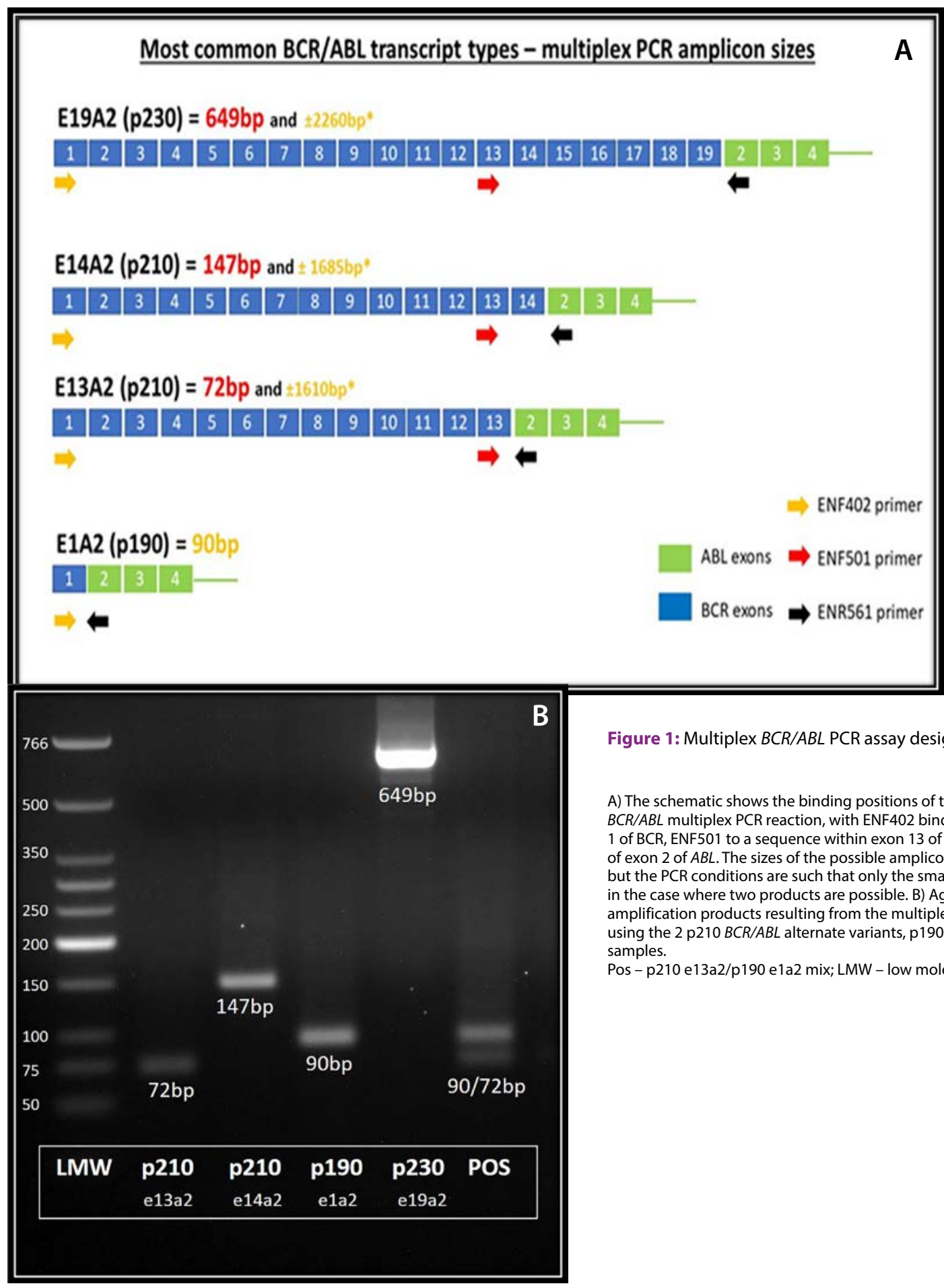

Figure 1: Multiplex $B C R / A B L P C R$ assay design

A) The schematic shows the binding positions of the three primers used in the $B C R / A B L$ multiplex PCR reaction, with ENF402 binding to a sequence within exon 1 of $B C R, E N F 501$ to a sequence within exon 13 of $B C R$ and ENR561 to the $5^{\prime}$ region of exon 2 of $A B L$. The sizes of the possible amplicon products are also indicated, but the PCR conditions are such that only the smaller amplicon will be amplified in the case where two products are possible. B) Agarose gel image showing the amplification products resulting from the multiplex $B C R / A B L$ variant assay when using the $2 \mathrm{p} 210 B C R / A B L$ alternate variants, p190 or p230 $B C R / A B L$ variants as RNA samples.

Pos - p210 e13a2/p190 e1a2 mix; LMW - low molecular weight marker 


\section{A: Specific BCR/ABL variant amplification products}

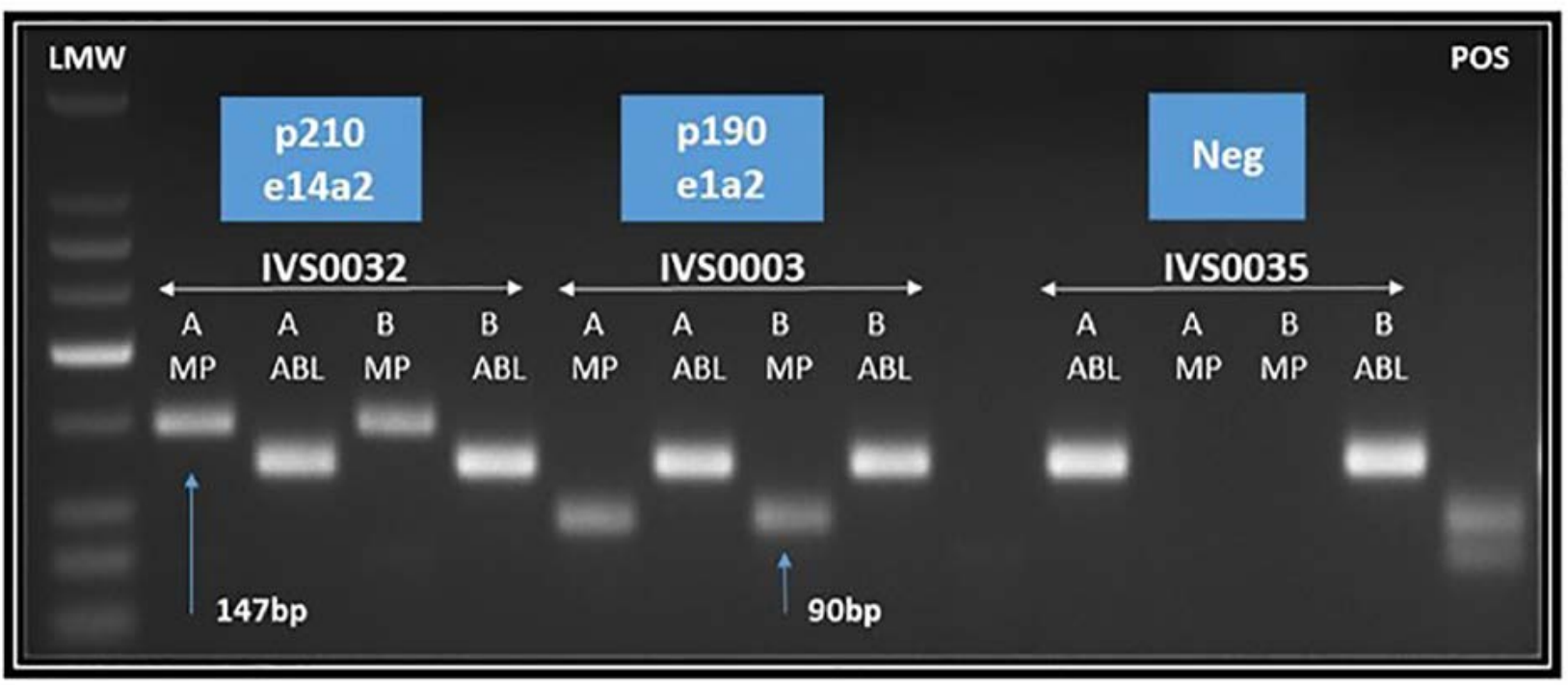

$B$ Sequence identification of multiplex BCR/ABL amplicons

A: Raw sequence data from patient CML3529- multiplex amplicon (e14a2)

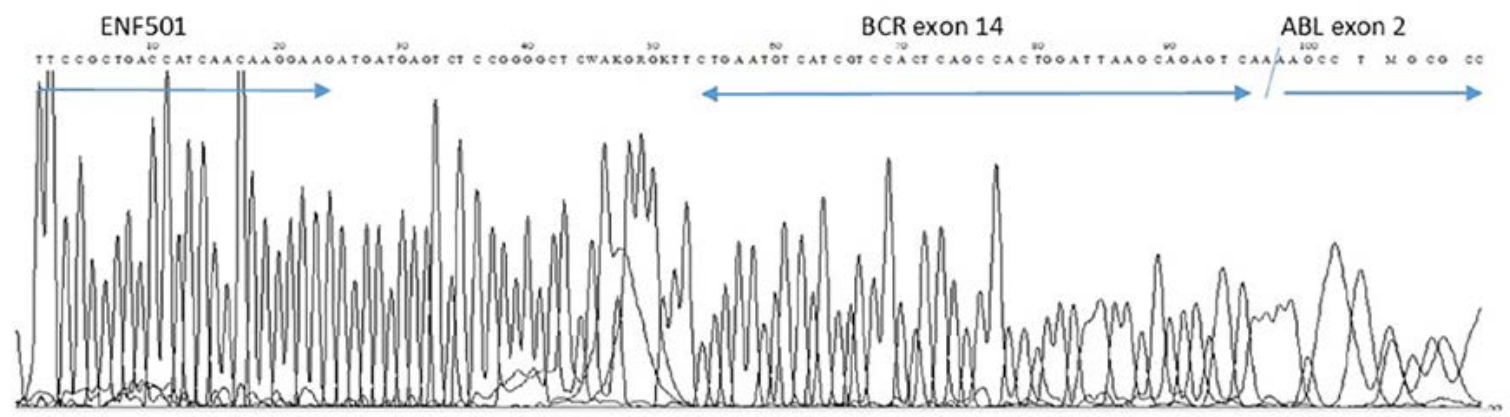

B: Raw sequence data from patient p190-87-multiplex amplicon (e1a2)

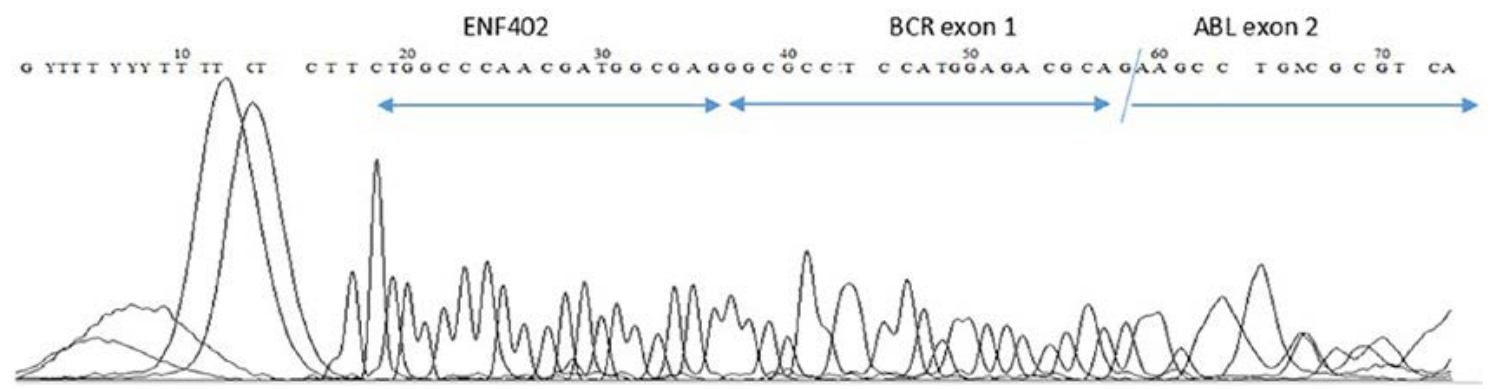

\section{C: Raw sequence data from patient MUT117 - multiplex amplicon (e19a2)}

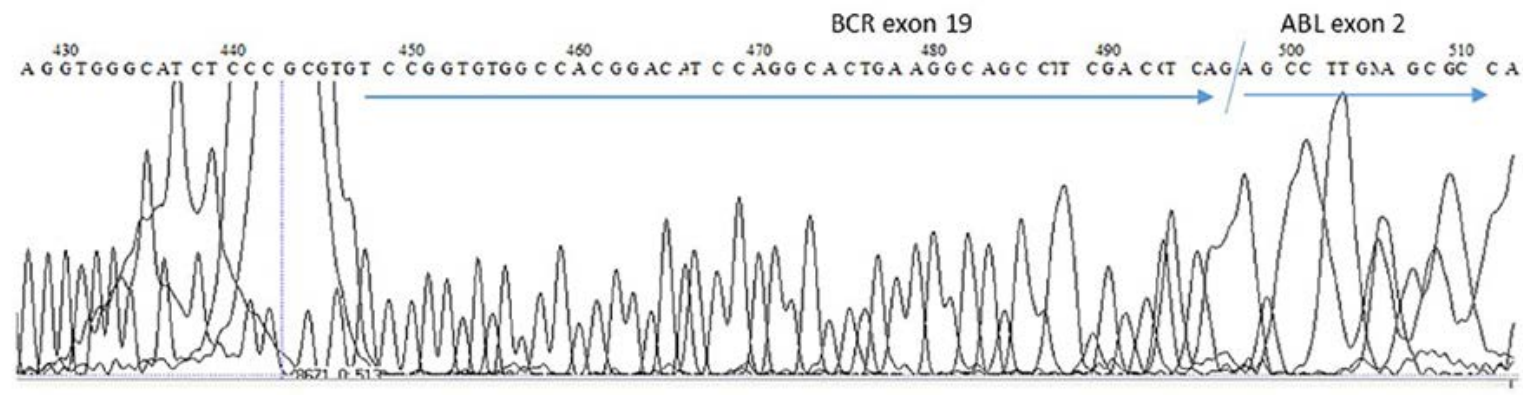

Figure 2: Specificity of the multiplex RT-PCR $B C R / A B L$ transcript variant assay

A) Agarose gel stained with ethidium bromide showing the multiplex analysis of $2 B C R / A B L$ positive RNA samples (IVS0032 and IVS0003), as well as a known $B C R / A B L$ negative sample (IVS0035). Two RT reactions are performed for each sample ( $\mathrm{A}$ and $\mathrm{B}$ ) and then $2 \mathrm{PCR}$ reactions are performed on each RT reaction, namely MP - BCR/ABL multiplex and $A B L$ - amplification of $A B L$ as an RNA integrity control. The blue boxes indicate the transcript calling based on the size of the MP amplicon sizes. Pos control - p210 e13a2/p190 e1a2 mix; LMW - low molecular weight marker. B) Sequences generated from the multiplex RT-PCR BCR/ABL amplicons were sequenced using primer ENR561 and resulting sequences aligned to the $B C R$ and $A B L$ reference sequences (NM_004327.5 and NM_005157.5 respectively), with the raw data shown here annotated to indicate the specific $B C R$ exon fused to exon 2 of $A B L$. 
with the CDNA as the input sample, and $A B L$ amplification acting as an RNA integrity control. Following the PCR reaction, the amplicons are separated on an agarose gel to identify the transcript variant based on the resulting amplicon size. Figure 1 shows the binding positions of the three primers in the $B C R /$ $A B L$ multiplex reaction and the expected amplicon sizes of the various transcript variants.

\section{Assay specificity}

To confirm that the assay only produces amplicons from RNA samples containing $B C R / A B L$ transcripts and that specific amplicon sizes correlate to the correct $B C R / A B L$ transcript variants, commercial RNA samples and RNA extracted from $B C R / A B L$-negative cell lines were analysed using the multiplex assay, with any resulting amplicons being sequenced to identify the corresponding translocation points. Figure $2 \mathrm{~A}$ shows the different amplicons produced from two known $B C R / A B L-$ positive transcript variants, as well as the lack of amplification with a known $B C R / A B L$-negative RNA. Figure $2 \mathrm{~B}$ shows the sequence analysis of several of the amplicons produced from this analysis, with break-points identified by comparison to the $N C B I$ reference sequences for $B C R$ and $A B L$. In total, nine different
RNA types were analysed, with no amplicons being produced for IVS0035, human testis, Jurkat, HL60 and RPMI8226 as expected and amplicons sized as e1a2 (90 bp), e13a2 (72 bp), e14a2 (147 bp) and e19a2 (650 bp) variants producing sequences that corresponded with the expected translocation points (i.e: $B C R$ exon 1 fused to $A B L$ exon 2 for e1a2). In combination with the analysis of EQA samples described below, the results of this exercise confirmed the specificity of the assay.

\section{Assay accuracy}

NEQAS EQA samples from five separate "AML translocation identification trials" were analysed in a blinded study using the multiplex RT-PCR $B C R / A B L$ assay (10 separate samples in total) to determine the accuracy of variant calling. Figure 3 shows the $B C R / A B L$-specific amplicons produced from the analysis of five of these samples and the corresponding variant call based on the amplicon sizing, while Table II shows the comparison between results obtained (in duplicate) and those reported by NEQAS for all 10 samples. Full correlation was observed between the multiplex results and those officially reported by NEQAS.

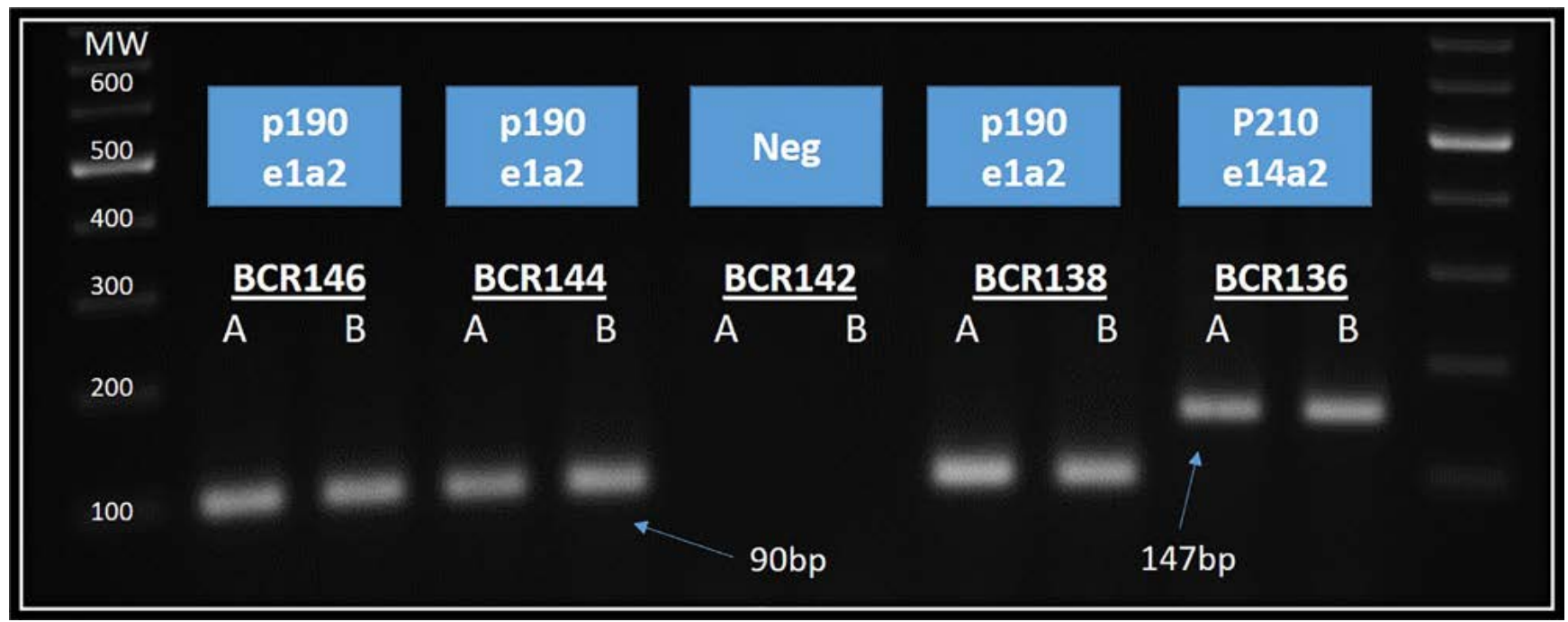

Figure 3: Multiplex RT-PCR BCR/ABL transcript variant assay assessment of NEQAS EQA samples

Agarose gel stained with ethidium bromide showing the amplicons produced from the assessment of 5 EQA samples. Two RT reactions are performed for each EQA sample $(A$ and $B$ ) and then $2 \mathrm{PCR}$ reactions are performed on each RT reaction, namely $B C R / A B L$ multiplex and amplification of $A B L$ as an RNA integrity control. Only the $B C R / A B L$ amplification results are shown here. The blue boxes indicate the transcript calling based on the size of the $B C R / A B L$ amplicon sizes. MW - $1 \mathrm{~kb}$ molecular weight marker.

Table Il: Comparison of $B C R / A B L$ variant calling using multiplex assay to reported NEQAS EQA report

\begin{tabular}{|c|c|c|c|c|c|}
\hline $\begin{array}{c}\text { EQA sample } \\
\text { name }\end{array}$ & $\begin{array}{c}\text { Multiplex amplicon } \\
\text { size (bp) }\end{array}$ & $\begin{array}{c}\text { Multiplex BCR/ABL } \\
\text { variant call }\end{array}$ & NEQAS EQA & $\begin{array}{l}\text { NEQAS trial } \\
\text { number }\end{array}$ & $\begin{array}{c}\text { Consensus } \\
\text { achieved }\end{array}$ \\
\hline BCR136 & $A$ and $B^{\#}: 147$ & p210-e14a2 & BCR/ABL pos- e14a2/p210 & 151602 & Yes \\
\hline BCR138 & $A$ and $B: 90$ & p190-e1a2 & BCR/ABL pos- e1a2/p190 & 151603 & Yes \\
\hline BCR142 & A and B: none & $\mathrm{BCR} / \mathrm{ABL}$ negative & No $B C R / A B L$ & 161702 & Yes \\
\hline BCR144 & $A$ and $B: 90$ & p190-e1a2 & BCR/ABL pos- e1a2/p190 & 161703 & Yes \\
\hline BCR150 & A and B: none & $\mathrm{BCR} / \mathrm{ABL}$ negative & No $B C R / A B L$ & 171803 & Yes \\
\hline BCR152 & A and B: 147 & $\mathrm{p} 210-\mathrm{e} 14 \mathrm{a} 2$ & BCR/ABL pos- e14a2/p210 & 181901 & Yes \\
\hline BCR154 & $A$ and $B: 90$ & p190-e1a2 & BCR/ABL pos- e1a2/p190 & 181902 & Yes \\
\hline BCR156 & A and B: none & $\mathrm{BCR} / \mathrm{ABL}$ negative & No $B C R / A B L$ & 181903 & Yes \\
\hline BCR158 & $A$ and $B: 90$ & p190-e1a2 & BCR/ABL pos- e1a2/p190 & 192001 & Yes \\
\hline
\end{tabular}

$\#-A$ and $B$ represent duplicate analysis 


\section{Assay sensitivity}

While this assay was designed to be used primarily at diagnosis to determine the $B C R / A B L$ transcript variant, it is possible that queries regarding the isoform type may be made several weeks or months into TKI therapy, during which time the levels of transcript may have been reduced by several logs. It may also be requested when cytogenetic results are not available, thus aiding in the initial diagnosis of $C M L$ or $\mathrm{Ph}+\mathrm{ALL}$ and $\mathrm{AML}$. For these reasons it is important to know the limit of detection of the assay to be able to report relevant qualitative pos/neg results. For this assessment $B C R / A B L$ p210-positive samples (e14a2 and e13a2) that have been previously quantified using our accredited $B C R /$ $A B L$ quantitative QRTPCR assay (NHLS) and covered a 4 log range of disease levels (43-0,005\%) were analysed by the multiplex RT-PCR BCR/ABL assay. Figure 4 shows the amplicons produced from one of the repeat assessments of the sample set.

The results of this analysis showed that p210 transcripts could be reproducibly and unequivocally detected at levels as low as $0.05 \%$, which allows for detection of transcripts over at least a 3 log range, therefore including patients who have achieved a complete cytogenetic response whilst on TKI therapy. Detection of levels below this gave inconsistent results between replicates (see Figure 4). It is important to note that as this is an endpoint $\mathrm{PCR}$ analysis, the $B C R / A B L$ amplicon band intensities are not necessarily related to the amount of starting material and thus cannot be used for quantification in any way (i.e: $0.050 \%$ vs $3.977 \%$ in Figure 4).

\section{Discussion}

$B C R / A B L$ is an oncoprotein that not only causes disease, but also fortuitously provides a target for a specific group of inhibitors known as TKIs. While there are still off-target effects, these are limited as the drugs can be designed as closely as possible to the unique nature of this specific cancer-protein, resulting in a very effective treatment for $\mathrm{CML}$ and $\mathrm{Ph}+$ acute leukaemias. Unfortunately, the $t(9 ; 22)$ translocation, which forms $B C R / A B L$, can occur at different breakpoints, resulting in several mRNA variants. While TKI therapy is not dependent upon the transcript type, ${ }^{15}$ highly sensitive MRD monitoring is transcript specific and false negative results will be obtained if the incorrect method is used. While the majority of CML patients express the common p210 BCR/ABL transcript variant and $\mathrm{Ph}+\mathrm{ALL}$ is associated primarily with the 190 mRNA variant, there are exceptions and these are unfortunately often missed. This can lead to a delay in diagnosis and inaccurate disease monitoring, all of which can affect patient outcome. This is fundamentally due to the lack of education of clinicians and pathologists regarding the limitations of the newer molecular techniques and the additional testing that is required.

Currently, using either commercial kits or in-house methods, it is possible to test for the different variants using separate qualitative RT-PCR methods, which can be performed in a sequential manner. However, this can be both costly and time consuming and clinicians are not always aware that alternate transcripts can be expressed. We thus aimed to develop a single assay that can simultaneously detect the vast majority of the reported $B C R / A B L$ transcript variants, ensuring that the correct monitoring method can be used at the start of therapy and that a diagnosis of variant $C M L$ or $B C R / A B L$ positive $A L L$ and $A M L$ is not missed. Our assay is capable of detecting all of the known $B C R / A B L$ variants that have breakpoints involving exon 2 of $A B L$ and any of the $B C R$ exons. This includes variants p210 (e13a2 and e14a2), p190 (e1a2) and p230 (e19a2). We validated the assay by testing it with a series of known $B C R / A B L$ positive and negative EQA samples, cell lines and commercial RNA and found the assay to be highly specific, accurate and reproducible. While the assay should preferably be used at diagnosis, the detection sensitivity limit showed that it is still capable of calling the correct variant when disease levels were as low as $0.05 \%$, when a patient has effectively just achieved a major molecular response to TKI therapy.

The testing algorithm for CML, AML and ALL cases should include karyotyping, so that the existence of the Ph chromosome can be established, as well as additional large DNA mutations. However this often fails due to technical issues and $\mathrm{t}(9 ; 22) \mathrm{FISH}$

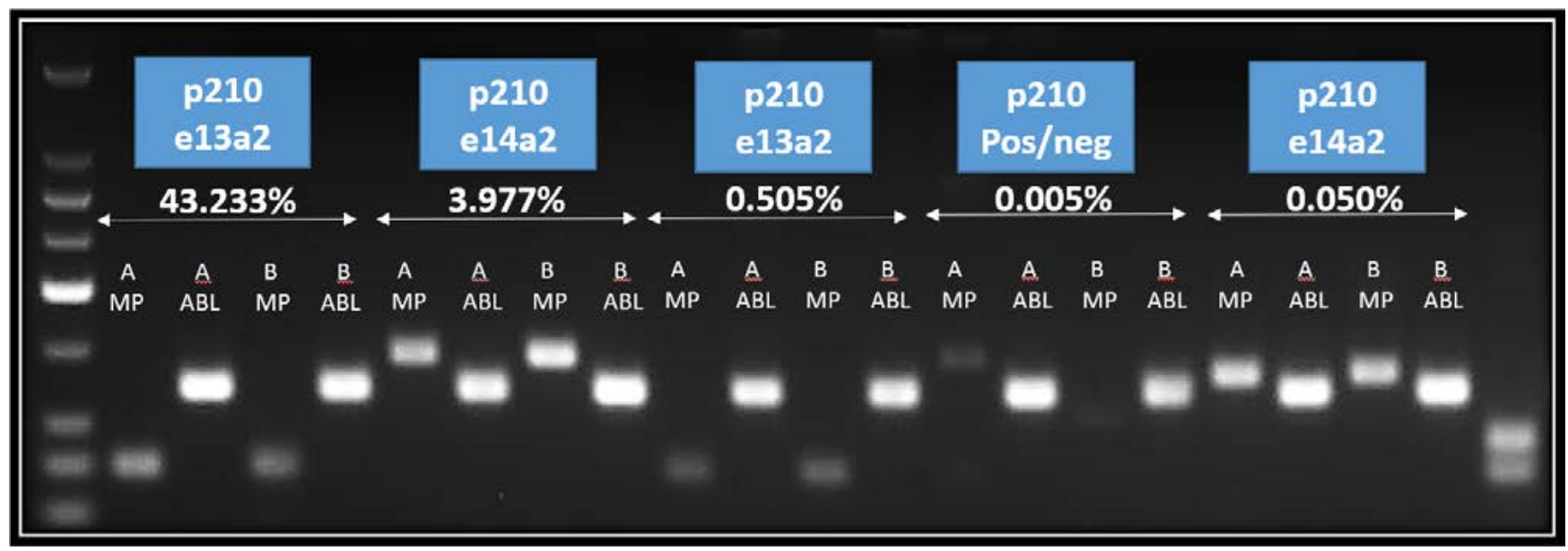

Figure 4: Multiplex RT-PCR $B C R / A B L$ assessment of $\mathrm{p} 210$ positive samples of various transcript loads

Agarose gel stained with ethidium bromide showing the amplicons produced using the multiplex assay. Two RT reactions were performed for each sample (A and $B$ ) and then 2 PCR reactions were performed on each RT reaction, namely MP - BCR/ABL multiplex and $A B L$ - amplification of $A B L$ as an RNA integrity control. The blue boxes indicate the transcript calling based on the size of the MP amplicon sizes. Pos control - p210 e13a2/p190 e1a2 mix; LMW - low molecular weight marker. 
is sometimes requested instead. If the $B C R / A B L$ translocation has been confirmed using either method, then it can be assumed that patients diagnosed with CML will be expressing the p210 transcript and monitoring via p210 QRTPCR can be initiated. However, if the p210 transcript is NOT detected at the first time point (usually within three months), it is imperative that the patient be tested for the expression of the alternate transcript. TKI responses in CML are relatively gradual and it is virtually impossible for a patient to have a $>4$ log response to TKI therapy within a three-month time frame, resulting in undetectable transcript. Continued testing using the p210 QRTPCR assay will result in the ineffective treatment monitoring of the patient, leading to possible haematological relapse without any warning, which may have dire consequences for the patient. We have, in fact, seen this exact scenario in two separate CML patients, one who was expressing the $\mathrm{p} 230$ variant and the other a $\mathrm{p} 190$ variant. In the cases where CML is suspected but cytogenetics is not available, then it is highly advised that the multiplex assay be used, instead of the p210 QRTPCR as a diagnostic test, to ensure that all possible $B C R / A B L$ variants are tested simultaneously for a definitive diagnosis. Thus far, using our multiplex BCR/ABL variant assay we have detected two $C M L$ patients expressing the p230 variant, four with p190 and a novel p210/p190 dual expression. Whilst this represents $<1 \%$ of the $\mathrm{CML}$ cases that we are monitoring through the p210 QRTPCR assay, which is in agreement with other studies, 8,9 another $6 \%$ (24/400) of cases continue to be regularly monitored by clinicians using this particular MRD assay despite never having the p210 variant confirmed, leaving these patients potentially at risk and possibly indicating a higher level of CML variants than usually reported.

$\mathrm{BCR} / \mathrm{ABL}$ positive $(\mathrm{Ph}+) \mathrm{AML}$ and $\mathrm{ALL}$ cases are considerably less common than $\mathrm{CML}$, however they can be treated with TKIs and monitored using QRTPCR assays, thus transcript identification is required. Similar to other international reports, the few $\mathrm{Ph}+$ AML patients that we have tested have all been expressing p210 mRNA transcripts, and $20 \%$ of the $\mathrm{Ph}+\mathrm{ALL}$ cases have been $\mathrm{p} 210$ positive, with the remainder expressing $\mathrm{p} 190$ transcripts. Thus in the case of ALL cases, it is very important that $\mathrm{p} 190$ expression is not automatically assumed.

On a final note, while the optimal TKI response of CML patients expressing p210 transcripts has been very well described, with results being expressed on the International Scale (European Leukemia Network guidelines), ${ }^{16,17}$ the optimal response patterns of those patients expressing the alternate transcripts is poorly described. Although similar log reductions over time would obviously be preferable, exact optimal time frames have not been standardised. In addition, both CML and Ph+ acute leukaemia patients may not respond to TKI therapy appropriately due to the presence of $A B L$ kinase mutations, mutations that block the binding of the TKI. The ABL kinase domain from all of the $B C R / A B L$ transcript variants can however be sequenced and alternate TKI therapy administered if mutations are detected.

\section{ORCID}

\section{K Shires (iD https://orcid.org/0000-0001-8570-3714}

\section{References}

1. Deininger MWN, Goldman JM, Melo JV. The molecular biology of chronic myeloid leukemia. Blood. 2000;96(10):3343-3356. https://doi.org/10.1182/blood. v96.10.3343.

2. Daley GQ, Van Etten RA, Baltimore D. Induction of chronic myelogenous leukemia in mice by the P210bcr/abl gene of the Philadelphia chromosome. Science. 1990;247(4944):824-830. https://doi.org/10.1126/science.2406902.

3. Swerdlow S, Campo $\mathrm{E}$, Harris $\mathrm{N}$, et al. WHO classification of tumours of haematopoietic and lymphoid tissue: Chronic Myelogenous leukemia, BCR-ABL1 positive. International agency for research on cancer 4th edition. 2008;32-37.

4. Faderl S, Talpaz M, Estrov Z, Kantarjan HM. Chronic myelogenous leukemia: biology and therapy. Annals of Internal Medicine. 1999;131(3):207-219. https:// doi.org/10.7326/0003-4819-131-3-199908030-00008.

5. Gajendra S, Sahoo MK. Philadelphia-positive acute myeloblastic leukemia: A rare entity. Journal of Neoplasm. 2016;1:1. https://doi. org/10.21767/2576-3903.100002.

6. Pui $\mathrm{CH}$, Evans WW. Treatment of acute lymphoblastic leukemia. New England Journal of Medicine. 2006;354:166-178. https://doi.org/10.1056/NEJMra052603.

7. Quintás-Cardama A, Cortes J. Molecular biology of bcr-abl1-positive chronic myeloid leukemia. Blood. 2009;113(8):1619-1630. https://doi.org/10.1182/ blood-2008-03-144790.

8. Gong Z, Medeiros LJ, Cortes JE, et al. Clinical and prognostic significance of e1a2 BCR-ABL1 transcript subtype in chronic myeloid leukemia. Blood Cancer Journal. 2017;7:e583. https://doi.org/10.1038/bcj.2017.62.

9. Verma $D$, Kantarjian $H M$, Jones $D$, et al. Chronic myeloid leukemia $(C M L)$ with P190BCR-ABL: analysis of characteristics, outcomes, and prognostic significance. Blood. 2009;114(11):2232-2235. https://doi.org/10.1182/blood-2009-02-204693.

10. Ottman OG, Pfeifer H. Management of Philadelphia chromosome-positive acute lymphoblastic leukemia (Ph+ ALL). Hematology Am Soc Hematol Educ Program. 2009;2009(1):371-381. https://doi.org/10.1182/asheducation-2009.1.371.

11. Shao $X$, Chen $D, X u P$, et al. Primary Philadelphia chromosome positive acute myeloid leukemia - a case report. Medicine. 2018;97(44):e12949. https://doi. org/10.1097/MD.0000000000012949.

12. Gabert J, Beillard E, van der Velden V, et al. Standardization and quality control studies of 'real-time' quantitative reverse transcriptase polymerase chain reaction of fusion gene transcripts for residual disease detection in leukemia - a Europe against cancer program. Leukemia. 2003;17:2318-2357. https://doi. org/10.1038/sj.leu.2403135.

13. Beillard E, Pallisgaard N, Van der Velden VHJ, et al. Evaluation of candidate control genes for diagnosis and residual disease detection in leukemic patients using 'real time' quantitative reverse-transcriptase PCR (RQ-PCR) - a Europe against cancer program. Leukemia. 2003;17:2472-2486. https://doi.org/10.1038/ sj.leu.2403136.

14. Branford S, Cross NCP, Hochhaus A, et al. Rationale for recommendastions for harmonizing current methodology for detecting BCR-ABL transcripts in patients with chronic myeloid leukaemia. Leukemia. 2006;20:1925-1930. https://doi. org/10.1038/sj.leu.2404388.

15. Hughes T, Deininger M, Hochhaus $A$, et al. Monitoring CML patients responding to treatment with tyrosine kinase inhibitors: review and recommendations for harmonizing current methodology for detecting $B C R-A B L$ transcripts and kinase domain mutations and for expressing results. Blood. 2006;108(1):28-37. https:// doi.org/10.1182/blood-2006-01-0092.

16. Baccarani M, Deininger NW, Rosti G, et al. European LeukemiaNet recommendations for the management of chronic myeloid leukemia: 2013 Blood. 2013;122(6):872-884. https://doi.org/10.1182/blood-2013-05-501569.

17. Baccarani M, Castagnetti F, Gugliotta G, Rosti G. A review of the European LeukemiaNet recommendations for the management of CML. Annals of Hematology. 2014;94:141-147. https://doi.org/10.1007/s00277-015-2322-2. 